\title{
NI ÁNGELES NI DEMONIOS: ELIZABETH GASKELL Y SUS PERSONAJES FEMENINOS
}

\author{
NEITHER ANGELS NOR DEVILS: ELIZABETH GASKELL AND HER FEMALE \\ CHARACTERS
}

Blanca Puchol Vázquez

Universidad Complutense de Madrid

\section{RESUMEN:}

El objetivo de este trabajo es analizar brevemente la figura de Elizabeth Gaskell, novelista del siglo XIX, y su obra, concretamente sus personajes femeninos. Dicho análisis se va a llevar a cabo en relación con la situación social que vivieron las mujeres de la época, cuestión que preocupó a muchos, entre ellos a la propia Gaskell.

\section{Palabras clave:}

Elizabeth Gaskell, cuestión de la mujer, ángel del hogar, mujer caída

\section{Abstract:}

The aim of this work is to briefly analyze Elizabeth Gaskell's life and work, especially her feminine characters. We are going to carry out this analysis regarding to the Woman Question, an issue which was of especial interest along de Victorian period, to which Elizabeth Gaskell belonged.

\section{KeYwORDS:}

Elizabeth Gaskell, Woman Question, angel in the house, fallen woman 
A lo largo de este artículo se va a analizar brevemente la figura de Elizabeth Gaskell, novelista del siglo XIX, y su obra, concretamente sus personajes femeninos, y se va a realizar este análisis dentro del marco social de la cuestión de la mujer y su situación en la Inglaterra decimonónica. Elizabeth Gaskell mostró gran preocupación por la situación social de las mujeres de su tiempo y se sirvió de su producción literaria para poner de manifiesto, denunciar y criticar la situación y los problemas a que se enfrentaban sus congéneres. Aunque no participó activamente en ningún grupo feminista, sí estuvo relacionada con varias figuras destacadas y promotoras de estos movimientos y, además, apoyó algunas de las iniciativas promovidas por los mismos.

Es necesario aludir en primer lugar a la situación social en que se encontraban las mujeres victorianas. Así es interesante mencionar cómo la sociedad estaba dividida en dos espacios: el espacio privado, tradicionalmente atribuido a la mujer y todo lo relacionado con lo femenino; y el espacio público, directamente relacionado, además de reservado, con el hombre y lo masculino.

\begin{abstract}
No en todas las épocas y sociedades lo privado y lo público han tenido las mismas
connotaciones que en la actualidad; sin embargo, con todas las salvedades y matices connotaciones que se pueden hacer con toda pertinencia, estimo [...] que lo privado y lo público constituyen lo que podríamos llamar una invariante estructural que articula las sociedades jerarquizando los espacios: el espacio que se adjudica al hombre y el que se adjudica a la mujer. A pesar de sus evidentes diferencias históricas esta distribución tiene unas características recurrentes: las actividades socialmente más valoradas, las que tienen un mayor prestigio, las realizan prácticamente en todas las sociedades conocidas los varones. [...] las que configuran o constituyen el espacio de lo público [...] Por el contrario, las actividades que se desarrollan en el espacio privado, las actividades femeninas, son las menos valoradas socialmente, [...] son las que no se ven ni son objeto de apreciación pública [ . . [ (Amorós1994:
\end{abstract}

Además de esta división socio-espacial, en la Inglaterra Victoriana surgióla dicotomía ángel-demonio, donde la figura del ángel del hogar se convirtió en el estereotipo de virtud y saber estar femeninos al que toda mujer debía aspirar. En contraposición estaban aquellas mujeres caídas en desgracia para la sociedad, las fallen women, aquellas que, en oposición a la figura del ángel eran consideradas demonios.

[...] The women in an urban space presented Victorian society with many complex social and moral problems. The modern urban life of women was the basis for the idea of the fallen woman. Respectable women, it was claimed, could not be par of the public sphere of city life. If women left the safety of the home and were on the streets, it was claimed, they became corrupted by the transgressive values of the city. They would be thought to be prostitutes or vulnerable workingwomen both victims of hostile and threatening environment. [...] Representing economic, social and moral deviance, a prostitute transgressed all expectations of feminine respectability. [...] It was for these reasons prostitution posed such danger to the social ideal of the passive dependent family-oriented female (Moore 2008).
Además, hay que mencionar el hecho de que la vida de las mujeres decimonónicas estaba ligada a la de los hombres. Es decir, encontrar un buen marido a quien cuidar y darle hijos era aquello a lo que debía aspirar toda mujer victoriana. Su labor era la de cuidar al marido y a sus hijos, encargarse del hogar y convertirlo en un lugar confortable y acogedor para el esposo, figurar como un adorno más de la casa ante las visitas, siempre comedida, siempre callada... En resumidas cuentas, y como apunta Joan Perkin en su libro, debían ser auténticos ángeles de su hogar:

Men's idea was of a decoratively idle, sexually passive woman, pure of heart, religious and self-sacrifying. The most popular image was of an 'angel in the house', an ivy-like wife who was also doting and self-abnegating mother, clinging to her husband on whom she was totally dependent (Perkin 1993: 86).

A esto habría que añadir el hecho de que las mujeres victorianas dependían por completo del esposo ya que, de tener algún tipo de posesión material esta pasaba a pertenecer al marido tras el matrimonio, lo mismo ocurría con el dinero, ya fuera heredado o adquirido mediante su trabajo. Fuera como fuese, la mujer no tenía ningún derecho sobre sí misma, sus posesiones o su sueldo, si lo tenía, ya que, por ley, al contraer matrimonio, tanto ella como sus posesiones, pasaban a ser propiedad del marido.

By marriage, husband and wife became one person in law - and that person was he. He had almost complete control over her body, and their children belonged to him. Unless a marriage settlement arranged things differently, the husband was entitled to all his wife's property and he could claim any money she earned. Indeed, as soon as a woman accepted a propos
to the man (Perkin 1993: 73-74).

A pesar de lo estricta que fue la sociedad victoriana, y la posición que las mujeres, en general, ocuparon dentro de la misma, hubo mujeres que no terminaron de adaptarse a estos parámetros. Ejemplo de ello es el caso que ocupa a este estudio pues, aunque Gaskell fue una feliz madre y esposa, dedicada al cuidado de los suyos y su hogar, también fue una mujer independiente y de carácter que se ocupó de sus propios asuntos y gestionó personalmente tanto sus contratos como sus ingresos. Además, fue capaz de compaginar sus labores literarias con sus obligaciones tanto domésticas como sociales, propias de la esposa de un ministro de la Iglesia Unitaria. Si bien es verdad que Gaskell ha sido tradicionalmente considerada una mujer conservadora y defensora de los estándares patriarcales victorianos, un ejemplo de ángel del hogar, igual de cierto es que pueden encontrarse ejemplos de acontecimientos y actitudes de la novelista a lo largo de su vida, además de críticas y denuncias de la situación de las mujeres en sus escritos, que hacen posible afirmar que quizás Gaskell no fuera tan conservadora como puede parecer a primera vista. Ejemplo de ello son algunos de 
los comentarios que pueden encontrarse en sus cartas donde, como muestra Perkin critica actitudes de su esposo o comenta la imposibilidad de las leyes, en su opinión, de proteger a las mujeres de sus cónyuges:

Gaskell For abominations such as these nothing but a change in the law would help though Elizabeth feared that even legislation could not protect a wife's property, writing to her friend Tottie Fox on Christmas Day, 1856 '... a husband can coax wheedle, beat or tyrannize his wife out of something and no law whatever will help that I can see' (Perkin 1993: 90).

A lo largo del siglo XIX fueron surgiendo diversos movimientos de mujeres que lucharon por cambiar la situación de la mujer: liberar a la mujer con respecto del marido; lograr el acceso de las mujeres a una educación mejor y más amplia, que fuera más allá de sus futuras labores como esposas, así como el acceso a estudios superiores; fundaron sociedades y escuelas para mujeres; lucharon por los derechos de la mujer trataron de cambiar la ley matrimonial vigente en el momento... Algunas de las figuras relevantes del movimiento proto-feminista fueron Anna Jameson, Adelaide Procter, Barbara Bodichon, Bessie Parkes, Florence Nightingale y Frances Cobbe. Elizabeth Gaskell, por su parte, no participó activamente en estos grupos pero fue amiga de muchas de estas mujeres y apoyó algunas de sus iniciativas como fue, por ejemplo, la firma de la petición, enviada al Parlamento en 1856, para cambiar la ley matrimonialvigente en el momento.

\section{The unlikely heroine who began the campaign for the reform was Barbara Leigh Smith (later Bodichon) [...] She published in 1854 her Brief Summary, in Plain Language, of the Most Important Laws of England concerning Women, pointing out how severely legally handicapped married women were. [...] A petition for change was sent to both Houses of Parliament in 1856, signed by 3,500 women including Elizabeth Barrett Browning, Jane Carlyle, Elizabeth Gaskell, Mary Howitt and Harriet Martineau. Seventy other similar petitions were signed by 24,000 women (Perkin 1993: 90)}

Hay que destacar también el apoyo y defensa, por parte de Gaskell, de las sociedades de mujeres así como la importancia que da en sus obras a la relación de amistad y apoyo entre mujeres.

Llama también la atención el hecho de que en una época en la que las mujeres no tenían control sobre sus finanzas, ni podían realizar transacciones sin la autorización del marido, Gaskell, quien no solo gestionaba y negociaba personalmente sus contratos y derechos con los editores sino que, además, administraba sus propias ganancias, logró comprar una casita de campo y amueblarla por completo sin el conocimiento, y por tanto sin la aprobación, de su marido, William, ya que su intención era que fuera una sorpresa para este último. Si bien es verdad que contó con la ayuda de sus yernos, del abogado y amigo de la familia William Shaen, y de George Eliot, su editor, esto no debe restar valor al hecho de que Elizabeth se las ingeniara para llevar a cabo todas las gestiones necesarias sin que su marido se enterara.

Por otro lado está el tema de la educación recibida por las mujeres de la época asunto que preocupó enormemente a Gaskell, quien consideraba que la formación recibida por las mujeres de su tiempo era insuficiente. Hay que destacar el hecho de que la educación recibida por Gaskell no se ajustó a los estándares de la época para una mujer. Esto fue así gracias, entre otras cosas, a la pertenencia de Elizabeth y su familia a la Iglesia Unitaria, movimiento religioso muy interesado en asuntos culturales, entre otros, y más aperturista, flexible y tolerante que la Iglesia establecida en cuanto a estos temas se refiere. Pueden encontrarse ejemplos en Cranford, donde se pone de manifiesto la falta de formación recibida por las mujeres cuando Miss Matty pierde todo su dinero y precisa buscar un trabajo; en Cousin Phillis, donde Gaskell presenta, por el contrario, a una joven dedicada al estudio; o en Wives and Daughters donde Molly Gibson, protagonista de la novela, trata de ampliar sus conocimientos a través de la lectura de libros de temática científica.

En cuanto a la presencia de la cuestión de la mujer en las obras de Gaskell, así como los distintos tipos de personajes femeninos que pueden encontrarse en las mismas, dos son las novelas que pueden venir a la cabeza, dado que su tema central gira en torno a las mujeres y su situación en la sociedad: Cranford y Ruth. Aunque en ambas novelas el tema de la mujer es central su tratamiento, además de la problemática presentada por su autora, es distinto en ambos casos. Así, en Cranford Gaskell muestra al lector un mundo gobernado por mujeres, en el que los personajes masculinos son bastante escasos. Además, pone de manifiesto las carencias que estas mujeres sufren como es, entre otros, la falta de educación y preparación para poder desarrollar una labor profesional, algo que se pone de manifiesto, como se ha comentado anteriormente, cuando tratan de decidir en qué puede trabajar Miss Matty para ganarse la vida tras la pérdida de todo su dinero a causa de la bancarrota del banco en que tenía depositados sus ahorros:

I thought of all the things by which a woman, past middle age, and with the education common o ladies fifty years ago, could earn or add to a living without materially losing caste; but at length I put even this last clause on one side, and wondered what in the world Miss Matty could do.

Teaching was, of course, the first thing that suggested itself. If Miss Matty could teach children anything, it would throw her among the little elves in whom her soul delighted. I ran over her accomplishments. [...]

No! there was nothing she could teach to the rising generation of Cranford, unless they had been quick learners and ready imitators of her patience, her humility, her sweetness, her quiet contentment with all that she could not do. I pondered and pondered until dinner was announced by Martha, with a face all blubbered and swollen with crying.

[...]But when the tea-urn was brought in a new thought came into my head. Why should not Miss Matty sell tea - be an agent to the East India Tea Company which 
then existed? I could see no objections to this plan, while the advantages were many - always supposing that Miss Matty could get over the degradation of condescending to anything like trade. Tea was neither greasy nor sticky - grease and stickiness being two of the qualities which Miss Matty could not endure (Gaskell 2009: 196-200)

Algunos críticos han destacado esta novela como presagio del movimiento feminista y lo hacen apoyándose en momentos como aquel en el que Deborah Jenkyns afirma que las mujeres no son iguales que los hombres, son superiores:

\begin{abstract}
Mrs. Gaskell presentation of the social conditions and currents of thought affecting the England of her day is able but limited: on the religio-scientific controversy, on educational reform, on the administration of justice, on political questions she has little or nothing to say. There is a mild foreshowing of the feminist movement in Cranford (1851), where it is said of Miss Deborah Jenkins, although she dressed in a cravat and a little bonnet-like jockey cap, which gave her altogether the appearance of a strong-minded woman, "she would have despised the modern idea of woman being equal to men. Equal - indeed! she 71-72).
\end{abstract}

Por otro lado, en Ruth el lector se encuentra ante el tema de la mujer caída: una joven que se deja llevar por sus sentimientos, que, al no contar con ningún tipo de apoyo ni directriz de una madre, se deja arrastrar y engañar por el hombre amado. Al huir y mantener una relación con él, sin estar casados, Ruth cae, socialmente, en desgracia. A lo largo de la novela, y a través de su trabajo, esfuerzo y perseverancia, Ruth logra poco a poco la redención. Sin embargo, la rígida sociedad victoriana ni perdona ni olvida fácilmente y, pese a los esfuerzos de la protagonista, la redención absoluta no es posible para ella salvo a través de la muerte la cual, irónicamente, llega de la mano de la misma persona que provocó su caída, siendo Bellingham, su antiguo amante, quien contagia a Ruth la enfermedad que le llevará a la muerte.

La mujer, su situación social, problemasy limitaciones, fueron temas que preocuparon a Gaskell a lo largo de toda su vida y que aparecen reflejados, de una u otra manera en todos sus escritos, y no sólo en los ya mencionados. Ejemplo de ello puede ser la presencia del tema de la infantilización de la mujer en Cousin Phillis:

This jarring breach in the Pickwickian geniality of closing chapters is seed of repressed anger about infantile status of women which is most finely explored in Cousin Phillis. Just as Peter sees his 50-year-old sister as a 'little Matty', so most of Elizabeth Gaskell's otherwise admirable men consign their sisters, wives and daughters to perpetual childhood (Stoneman, 1987: 98).

En esta novela Gaskell presenta ante el lector a una joven bien educada a la cual su padre ha introducido en interesado en el estudio del latín, la historia y la literatura, una joven aún niña desde el punto de vista de su feminidad, de la cual Phillis no es aún consciente al comienzo de la novela. Phillis vive en lo que parece una burbuja de vida ideal, consagrada al estudio, la familia y el trabajo, sin apenas contacto con la sociedad exterior, burbuja en la que la mantienen sus padres como un preciado tesoro, tratando de impedir que su pequeña sea contaminada por la sociedad, fomentando, de alguna manera, la infantilización de su hija. A pesar de los esfuerzos paternos, puede verse cómo Phillis llega poco a poco a la vida adulta, empiezan a interesarle pequeños detalles propios de las mujeres de la época como era el adorno de los vestidos y los sombreros, vanidades propias de una mujer adulta y que para nada preocupan a una niña de campo. Sin embargo Phillis crece y, al hacerlo, comienza a pensar y preocuparse por estas pequeñas vanidades:

[...] but the women are apt to tarry before coming to me, to put on ribbons and gauds; as if they could hear the message I bear to them best in their smart clothes. [...] Phillis, I am thankful thou dost not care for the vanities of dress!' Phillis reddened a little as she said, in a low humble voice-

'But I do, father, I'm afraid. I often wish I could wear pretty-coloured ribbons round my throat like the squire's daughters.' (Gaskell 2009: 277).

Las apreciaciones del narrador ayudan al lector a ver el cambio que Phillis sufre a lo largo de la novela, llamando la atención con comentarios como el siguiente, en el cual hace referencia a la venda en los ojos que parecen tener los padres de la joven.

So I went to the farm certainly, on an average, once a month during that autumn [...] I can only remember one small event, and that was one that I think I took more notice of than any one else: Phillis left wearing the pinafores that had always been so obnoxious to me; I do not know why they were banished, but one of my visits I found them replaced by little linen aprons in the morning, and a black silk one in the afternoon. And the blue cotton gown became a brown stuff one as winter drew on; [...] (Gaskell 2009: 280).

Quizás esto no parezca muy relevante pero son los primeros signos de que Phillis está cambiando y de cómo sus padres son incapaces de verlo. Solo el extraño, el que viene de fuera, lo percibe. Y lo mismo ocurre con los sentimientos que Holdsworth despierta en la joven, solo Paul se percata de la transformación de su prima, de los nuevos sentimientos que parecen aflorar en su interior. Todo ello es muestra de cómo poco a poco el exterior, la sociedad, se abre paso en esa pequeña burbuja que es la granja, y cómo lo hace a través de personajes que vienen de fuera como Paul Manning (narrador y primo de Phillis) y su jefe, el señor Holdsworth. Los sentimientos que este último despierta en Phillis son el culmen de la transformación de esta en mujer, frustrando definitivamente el intento del reverendo Holman (padre de Phillis) de mantener a su hija en una fase infantil permanente. 
En cuanto a las limitaciones que sufrieron las mujeres, tanto laboral como socialmente hablando, por el mero hecho de ser mujeres, cabría mencionar la novela Syliva's Lovers, donde Gaskell muestra la imposibilidad de Hester de ser nombrada socia del negocio por el mero hecho de ser mujer:

John Foster was fumbling with the papers a little nervously: Jeremiah spoke-

We have not thought it necessary to commend Hester Rose to you; if she had been a lad she would have had a third o' the business along wi' yo'. Being a woman, it's ill troubling her with a partnership; better give her a fixed salary till such time as she marries.

He looked a little knowingly and curiously at the faces of the young men he addressed. William Coulson seemed sheepish and uncomfortable, but said nothing, leaving it as usual to Philip to be spokesman.

'If we hadn't cared for Hester for hersel', master, we should ha' cared for her as being forespoken by yo'. Yo' and Master John shall fix what we ought $\mathrm{t}^{\prime}$ pay her and I think I may make bold to say that, as our income rises, hers shall too-eh, Coulson?' (a sound of assent quite distinct enough); 'for we both look on her as a sister and on Alice like a mother, as I told her only this very day.' (Gaskell 2004: $163)$

Pero Elizabeth Gaskell no solo muestra los problemas a que se enfrentaron las mujeres de su tiempo sino también la existencia de mujeres que, de alguna manera, se rebelaron, fueron un paso más allá. Como ejemplo puede mencionarse a Mary Barton, quien, por ejemplo y como afirma la doctora Durán, da el salto a la esfera pública a declarar en el juicio contra Jem:

[...] Gaskell makes a place for women in the public and political domain: during the trial, Jem's life is in Mary's hands, and she takes all kinds of risky actions improper for a woman to succeed. [...] So, Mary Barton, seemingly simply a factory girl, represents a powerful force who reasons, speaks and acts in the public world, protecting her father and rescuing her lover (Durán, 2006:473).

Otro ejemplo puede encontrarse en North and South, novela cuya protagonista, Margaret Hale, es una joven que, si bien no se rebela abiertamente contra el orden establecido tampoco encaja en el estereotipo de ángel del hogar. Margaret es una joven acomodada, de un pueblecito del sur de Inglaterra, que se ocupa del cuidado de sus padres y de su hogar. Tras su traslado a Milton (Manchester), ciudad industrial del norte, se encuentra ante un mundo completamente nuevo y opuesto a cuanto conoce, lo que le lleva a interesarse por conocer y entender tanto el mecanismo de la ciudad como a sus habitantes y las relaciones existentes entre unos y otros. A lo largo de la novela Margaret demuestra ser una joven de valía, mujer de carácter e independiente y autónoma en cuanto a sus actos se refiere. No se limita a estar callada y cosiendo, como sería de esperar en una joven victoriana, mientras los hombres conversan, sino que interviene en dichas conversaciones, dice su opinión en voz alta, discute en torno a temas económicos y políticos como, por ejemplo, el enfrentamiento existente entre obreros y patronos o las diferencias sociales entre ricos y pobres. Ejemplo de ello es la discusión que mantiene con el señor Thornton (empresario) en torno a la huelga que se avecina. Puede parecer esta una escena sin importancia desde el punto de vista actual sin embargo cobra importancia si se tiene en cuenta el hecho de que lo se esperaba de una mujer era, como ya se ha comentado anteriormente, que estuviera callada, cosiendo escuchando, que no tuviera ideas propias, menos aún que las expresara, y mucho menos que lo hiciera delante de un hombre.

Por otro lado habría que destacar también de esta novela el hecho de que sea Margaret quien salva a Thornton, y lo hace al menos en dos ocasiones. La primera tiene lugar cuando los enfurecidos obreros se dirigen a casa de Thornton, donde Margaret salva la situación al ser ella quien recibe la pedrada dirigida a Thornton al interponerse entre este y la muchedumbre. La segunda ocasión tiene lugar al final de la obra cuando, tras heredar cierta cantidad de dinero, propone a Thornton, entonces arruinado, ser socios. Si bien es cierto que esta escena termina siendo un tanto de corte romántico, también lo es el hecho de que Gaskell no reconcilia a ambos sexos ni permite un compromiso entre ambos hasta ahora, momento en que se enfrentan el uno al otro como iguales:

[...] So, only when Mr. Thornton's mill fails and he steps down from being a 'master'; only when Margaret has abandoned her chivalric past and engaged with the present, are they reunited. After hearing of his reforms and of his failure, she approaches Thornton as a partner in business; only then do they meet as equals, conscious of their faults (Duran, 2006: 479).

Puede decirse que Gaskell en esta novela subvierte el control y dominación masculina y enfatiza el poder femenino en un intento de igualar el mundo masculino y el femenino. Como se puede leer en el artículo de la doctora Durán: “[...] So, by writing this story of conciliation between classes and sexes through the influence and intervention of womanly values, Gaskell was exercising a peculiarly Victorian mode of womanly authority" (Durán, 2006:480).

Todos estos son solo algunos ejemplos de cómo la cuestión de la mujer fue un asunto muy presente en la Inglaterra decimonónica. Gracias a trabajos como los de Gaskell puede observarse hoy la idea general y estereotipada que tenían entonces de la mujer, su condición y su lugar dentro de la sociedad, así como el surgimiento de voces discordantes que trataron de cambiar el orden establecido. Gaskell muestra al lector un amplio abanico de personajes, no solo mujeres sino también hombres, que reflejan la variedad de opiniones y actitudes que fueron surgiendo en torno a esta cuestión, y describe mujeres que no terminan de encajar con los estereotipos victorianos, mujeres que no eran ángeles pero tampoco demonios. Todo ello da valor y confiere interés al estudio de sus obras. 


\section{REFERENCIAS BIBLIOGRÁFICAS}

Amorós, Celia, “Espacio público, espacio privado y definiciones ideológicas de 'lo masculino' y 'lo femenino'", en Amorós, Celia, Feminismo, igualdad y diferencia, México, UNAM, PUEG, 1994, pp. 23-52. Internet. 29-03-2016. http://emujeres.net/sites/ default/files/espacio_publico_espacio_privado_definiciones_ideologicas_masculino_ femenino_0.pdf

Durán, Isabel, "Elizabeth vs. Mrs Gaskell: the two voices of a Victorian feminist" $A$ Pleasure of Life in Words: A Festschrisft for Angela Downing / coord. por Marta Carretero et al., Vol. 2. Madrid, Departamentos de Filología Inglesa I y II, Universidad Complutense, D.L. 2006, pp. 463-482.

Gaskell, Elizabeth, Cranford / Cousin Phillis, London, Penguin, 2009.

Gaskell, Elizabeth, Mary Barton, London, Penguin, 2009.

Gaskell, Elizabeth, North and South, London, Penguin, 2003.

Gaskell, Elizabeth, Ruth, London, Penguin, 2004.

Gaskell, Elizabeth, Sylvia's Lovers, London, Penguin, 2004.

Hopkins, A. B., "Liberalism in the Social Teachings of Mrs. Gaskell”. Social Service Review, Vol. 5. No. 1 (Mar., 1931), pp. 57-73. The University of Chicago Press Internet. 26-09-2014. http://www.jstor.org/stable/30009643

Moore, Meredith, "Private vs Public: Female Sexuality in Victorian Culture". English 156 (History of Art 152) - Victorians in Togas: Classicism and Empire in Victorian Literature and Art, Brown University. Internet. 29-03-2016. http://www.victorianweb.org/ sculpture/nudes/1moore4.html

Perkin, Joan, Victorian Women, London, John Murray, 1994.

Stoneman, Patsy, Elizabeth Gaskell, Brighton, Harvester Press, 1987. 